\title{
Software-Based Encoder for UHD Digital Signage System
}

\author{
S.W. MOON*, J.W. LEE*, J.S. LEE*, H.T. JUNG*, K.S. YOON* \\ *ETRI(Electronics and Telecommunications Research Institute), Korea \\ moonstarry@etri.re.kr, jeongwoo@etri.re.kr,jslee2365@etri.re.kr, htjung@etri.re.kr, ksyoon@etri.re.kr
}

\begin{abstract}
With the rapid growth of the digital signage industry, high resolution video contents are used for digital signage. In this situation, advertisers want to use UHD video contents to advertise their product. In this paper, we propose a softwarebased encoder for UHD digital signage contents which can divide frames into appropriate form for the layout of a digital signage display system and manage quality of divided frames.
\end{abstract}

Keywords — Digital Signage, Software-Based Encoder, Ultra High Definition

\section{INTRODUCTION}

Since the digital signage industry has been widely propagated, advertisers want to use high resolution video contents to advertise their products. [1]-[4] For this reason, advertising producers need an encoder for Ultra High Definition(UHD) video contents to make UHD digital video signage. In general, hardware-based encoders are used to encode high resolution video contents. However, hardwarebased encoders are expensive and hard to customize. Furthermore, it is difficult to improve the performance of a hardware-based encoder without changing the machine. To solve these problems, we propose a software-based encoder for UHD digital signage system.[5]-[6]

To reduce the price of digital signage display system, multipanel based devices are used to display digital signage. Figure 1 shows the multi-panel based device which can display UHD video contents by using four full-HD panels. This means that an encoder for digital signage contents should convert the digital signage content to suit with multi-panel signage device.[7]-[9]

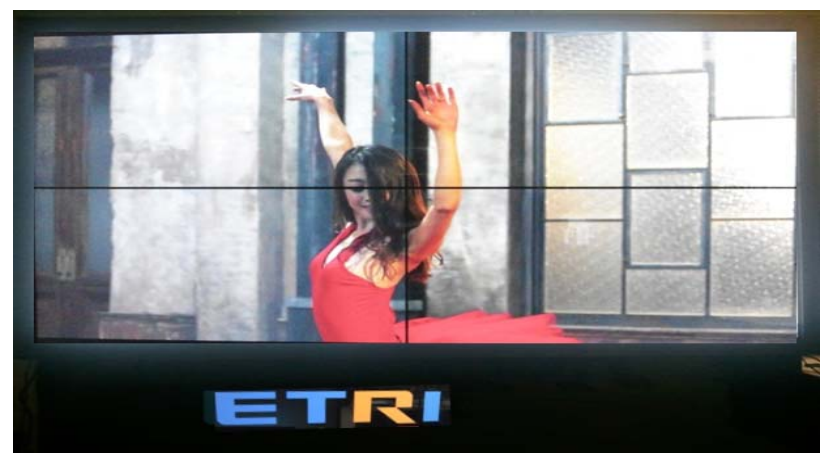

Figure 1. Multi-panel based device for digital signage

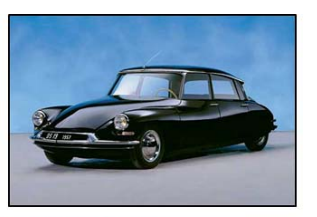

$3840 \times 2160$ @30fps Dual-LINK DVI, 1 port (300Mpixel/s) (a)

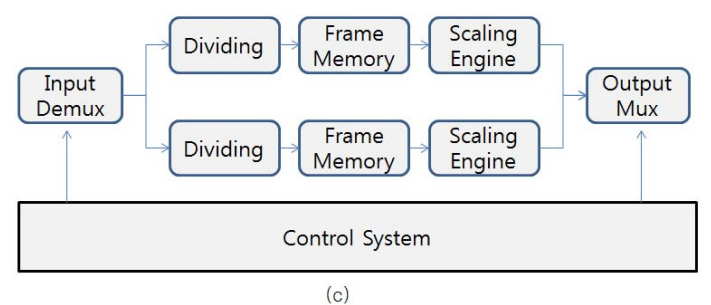

Figure 2. Concept design of Multi-panel based device for digital signage

In this paper, we propose an encoder which can divide a digital signage content and encode each content in parallel. If the encoded UHD digital signage content's quality is low, customers do not feel attractive from the digital signage contents whether the content's resolution is high or not.[5]-[7] The proposed encoder can encode UHD digital video signage contents with high quality and low bitrate.

The proposed method divides an UHD digital signage contents into pieces then divides each piece into slices. By using parallel encoding, we can encode the digital signage content appropriately for the layout of multi-panel device in short time. We can manage the quality of each piece of the digital signage content because we can set each piece's parameter individually.

This paper is organized as follows. We explain the proposed software-based digital signage encoder in section II. Experimental results are given in section III and concluding remarks are given in section IV.

\section{Proposed Software-based Encoder}

\section{A. Software-based Encoder Design}

Figure 3 shows the encoding processes of the proposed soft-ware based UHD digital video signage encoder. First, each frame of digital video signage contents is divided into 


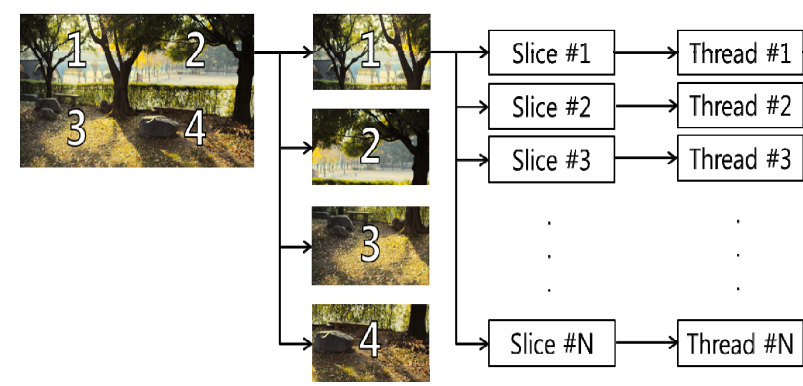

Figure 3. Design of the digital signage encoder

pieces to suit for the layout of digital signage display device. Each piece is also divided into slices which are encoded in parallel. A thread is given for a slice, so we can manage the number of slices which are used to encode a frame. Because of pieces in same frame are encoded in parallel, the proposed encoder can control parameters for each piece. If a piece's encoding quality is lower than other pieces, the encoder will enhance the quality of the piece. By using this method, we can normalize the quality of the target UHD digital video signage content.

Since the proposed encoder divides a frame into pieces, we can set each piece's encoding parameters like bitrate, framerate, size and motion estimation method individually. If an advertiser has different priority of digital signage contents, the advertiser can allocate more bitrate for important digital signage contents. When the encoding is finished, each slice is combined to reconstruct original frame. Because of the frame is combined after dividing process, blocking artifacts can be shown at the edge of slices. We use deblocking algorithm to remove blocking effect and get clear frame.

The proposed encoder which uses dividing encoding method is faster than a simple whole frame encoder. UHD video frames are bigger than cache memory. If we encode whole frame without dividing process, the frame data overflow cache memory and violate the memory which should be used for next frame. Since each divided slice's size is small, memory violation problem is solved by using dividing encoding process.

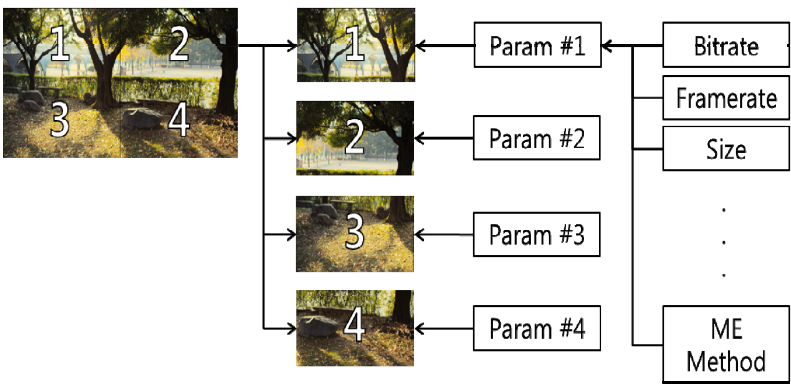

Figure 4. Encoding parameters of the digital signage content
TABLE 1. COMPARISON BETWEEN THE PROPOSED METHOD AND A SIMPLE IPP H.264 ENCODER

\begin{tabular}{|c|c|c|}
\hline & Proposed Method & IPP encoder \\
\hline Speed (fps) & 15.48 & 9.30 \\
\hline CPU usage (\%) & 96.58 & 99.27 \\
\hline
\end{tabular}

\section{B. Encoder Implementation}

We use H.264 codec for UHD digital signage contents. The H.264 format is the most popular codec for high resolution video contents and has high compression ratio. The H.264 standard also considers dividing frames into slices. We support main profile which is appropriate to generate high compressed video contents.[10]

The proposed encoder encodes target UHD digital video signage contents as follows. First, read a raw UHD digital video signage content with encoding parameters. We use YUV stream format for raw UHD digital video signage contents. The proposed encoder is created and initialized with given encoding parameters. Next, the input YUV stream is divided into pieces logically and each piece is divided into slices by considering number of thread. Each slice is encoded by single thread in parallel. After a frame is encoded, the proposed encoder gets $\mathrm{QP}$ of each piece to control next frame's quality. If specific piece's quality is too high or low, it will be adjusted in next frame.

Since digital signage contents can be transferred in online, the UHD digital signage video content's bitrate should be less than $30 \mathrm{Mbps}$. It means that the compression ratio of the target UHD digital video signage content should be very high. The proposed method can compress the raw YUV stream to $30 \mathrm{Mbps}$ H.264 video without degrading the resolution of digital signage contents.

\section{III.EXPERIMENTAL RESULTS}

We used two Intel Xeon X5680 processors to encode UHD digital video signage contents to evaluate the proposed method. The proposed method uses OpenMP technology for parallel processing. The resolution of videos which are used to

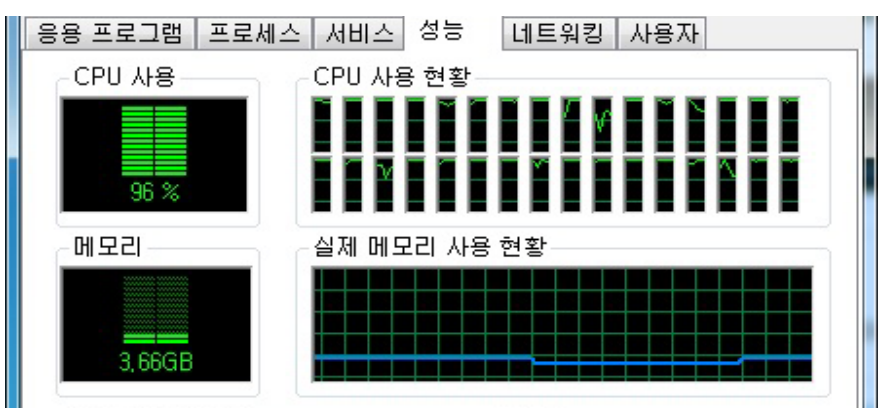

Figure 5. CPU usage of UHD digital signage encoder 

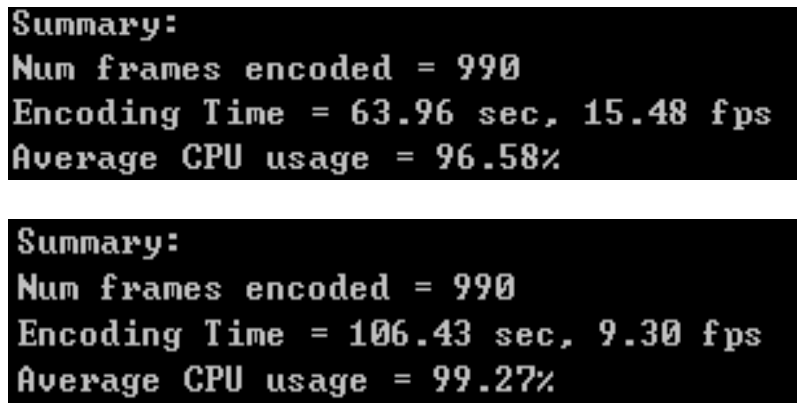

Figure 6. Comparison between the proposed method(top) and a simple IPP H.264 encoder(bottom)

evaluate the proposed has $3840 \times 2160$. We divided the target UHD digital video signage into four pieces and same initial encoding parameter is given for every piece. In this paper, we compare the simple H.264 encoder in IPP sample with the proposed software-based UHD digital signage encoder.

Table 1 and Figure 6 shows that the proposed method is 60 percentage faster than existing simple IPP H.264 encoder despite IPP encoder also supports multi thread processing. The proposed method is faster than existing method because the two-level parallel processing need less time to pipeline threads which encode slices.

Figure 7 shows that encoded UHD digital video signage contents which are encoded by using the proposed method have high fidelity after encoding. The average peak signal to noise ratio(PSNR) of encoded contents is over $45 \mathrm{~dB}$ and even the minimum value is over $35 \mathrm{~dB}$ while encoded contents are compressed to $30 \mathrm{Mbps}$ H.264 video.

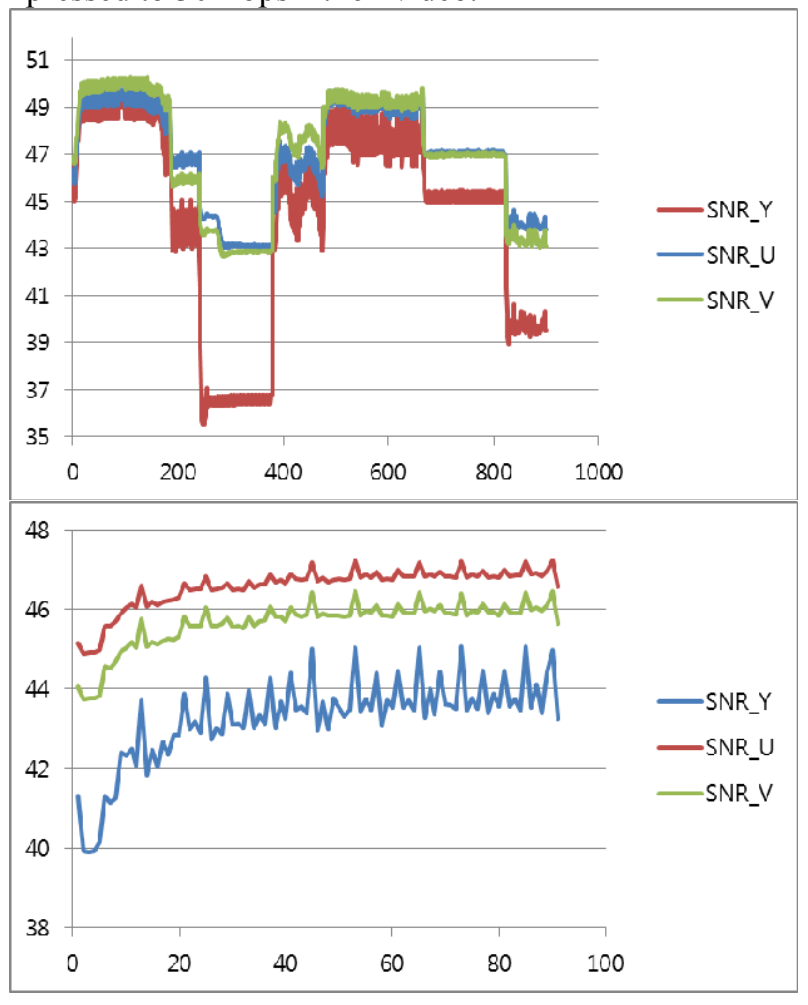

Figure 7. PSNR of encoded UHD digital video signage contents

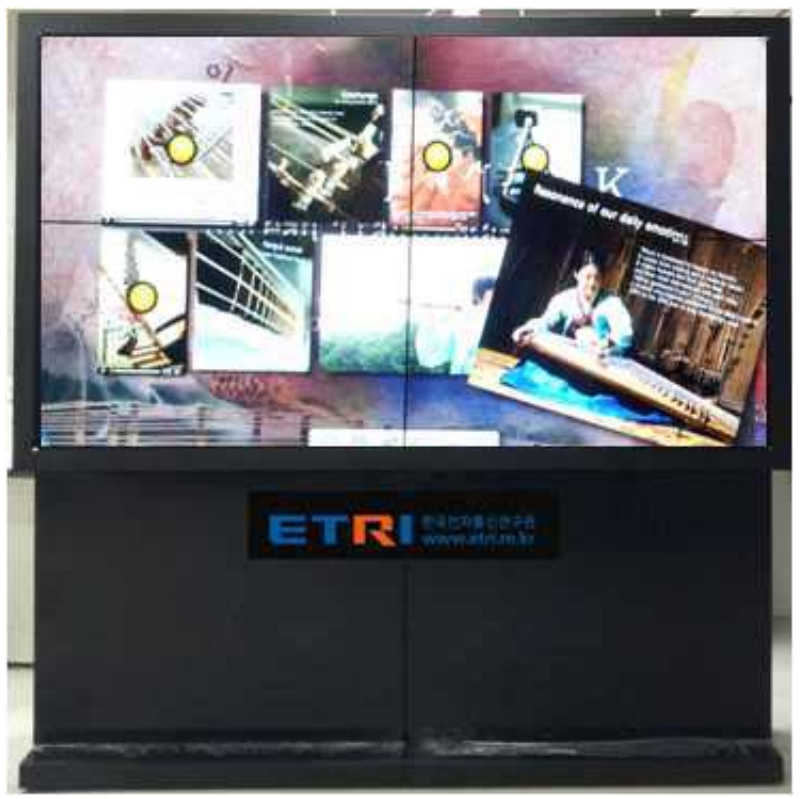

Figure 8. Multi-panel based digital signage display device

We also checked the fidelity based on human visual system by using multi-panel based digital signage display device with experts. The encoded UHD digital signage contents have no blocking artifact and have high fidelity. Since the encoder control each piece's quality, every panel's content have similar quality.

\section{IV.CONCLUSIONS}

In this paper, we proposed a software-based UHD digital signage encoder which can encode UHD digital video contents with high fidelity in short time by using parallel encoding technology. Our experimental results show that the proposed encoder guarantees better speed than an existing encoder on same system. In future research, we will try to use HEVC codec to reduce the bitrate of digital signage contents and encode 3D UHD video contents with the proposed encoder.

\section{ACKNOWLEDGMENT}

This work was supported by MSIP(Ministry of Science, ICT and Future Planning) (10041539 High Compression, Low Loss Content Creation/Distribution/Display Technology Development for 8K-Video Service).

\section{REFERENCES}

[1] Grimmett, Richard, et al. Pocket guide to the birds of the Indian subcontinent. C. Helm, 1999.

[2] ITU-T H.780(ex H.FDSS), Digital signage: Service requirements and IPTV-based architecture, Draft new Recommendation, June 2012.

[3] Steve G urley. "Trends in mobility and the implications on digital signage", DigitalSignageToday White paper. 2011

[4] Intellian systems(2011), "Digital Signage Content distribution system optimization technology", Digital signage news, vol.05.2011. SEP.

[5] C. Yoon, H. Lee, S. Hyun Jeon, and Hyunwoo Lee, "Mobile digital signage system based on service delivery platform location based 
targeted advertisement service," International Conference on ICT Convergence (ICTC), 28-30 Sept. 2011, pp. 582 - 586.

[6] I. Satoh, "An Agent-Based Framework for Context-Aware Digita Signage," in 1st International Symposium on Ambient Intelligence (ISAmI 2010), Guimaraes, Portugal, 2010, pp. 105-112.

[7] C. Bauer, P. Dohmen, C. Strauss, "Interactive Digital Signage-An Innovative Service and Its Future Strategies", in Proceeding EIDWT '11 Proceedings of the 2011 International Conference on Emerging Intelligent Data and Web Technologies, 2011, pp. 137-142.

[8] J. Muller, J. Exeler, M. Buzeck and A. Kruger, "Reflective Signs: Digital Signs That Adapt to Audience Attention," in $7^{\text {th }}$ International Conference Pervasive Computing (Pervasive 2009), Nara, 2009, pp. 17-24.

[9] J. Muller and A. Kruger, "MobiDiC: Context Adaptive Digital Signage with Coupons," in 3rd European Conference on Ambient Intelligence (AmI 09), Salzburg, 2009, pp. 24-33.

[10] Wiegand, Thomas, et al, "Overview of the H. 264/AVC video coding standard." in Circuits and Systems for Video Technology, IEEE Transactions on, vol.13.7, 2003, pp. 560-576.

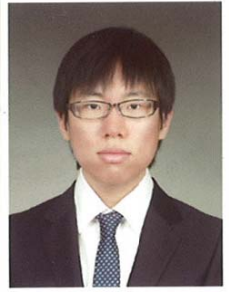

S.W. MOON(Korea, 1988. 06. 18.) received his B.S and M.S degrees of computer science from KAIST, Korea in 2010 and 2012. He is a RESEARCHER of Electronics and Telecommunications Research Institute(ETRI), Korea. His research interests are Digital Watermarking, Video Forensic, and Video Processing.

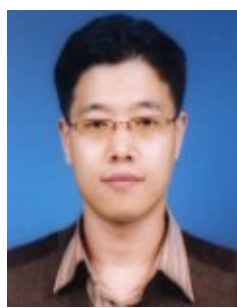

Jungsoo Lee received his B.S. and M.S. degrees from Jeonbuk University, Korea in 1995 and 1997, respectively and his $\mathrm{Ph} . \mathrm{D}$. degree in Electronic Engineering from Hanyang University, Seoul Korea in 2005. From 2000 to 2005, he was a senior member of MarkAny Research Institute. Currently, he is a senior member of Electronics and Telecommunications Research Institute(ETRI). His research interests are digital watermarking, fingerprinting, image processing, digital rights management, digital cinema and digital signage.

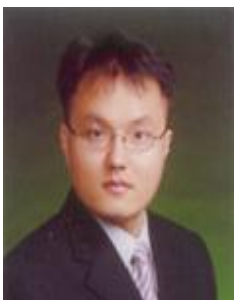

Jeongwoo Lee received the B.S. degree in information and telecommunication engineering from Jeonbuk National University, Jeonju, Korea, in 1996, and the M.S. degree in information and communication engineering from Gwangju Institute of Science and Technology (GIST), Gwangju, Korea, in 1998. He received the $\mathrm{Ph} . \mathrm{D}$. degree in the Information and Communications Department from GIST in 2003. He is currently working in Electronics and Telecommunications Research Institute (ETRI). His research interests include digital video coding algorithms, implementations for H.264 and HEVC, rate control algorithms for video coding, scalable video compression, and gpu-based coding algorithms.

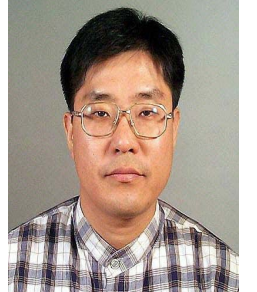

Hyo Taeg Jung received his B.E. and M.S. in Electronic Engineering at Kyungpook National University and Computer Science at Yonsei University, Korea in 1986 and 1997, respectively. He received his $\mathrm{Ph} . \mathrm{D}$. degree in Software Engineering at the University of Texas at Dallas in 2008. Since 1987, he has been working as a principal researcher at Electronics and Telecommunications Research Institute(ETRI). His research interests are requirement engineering, contents engineering, digital content management and digital signage.

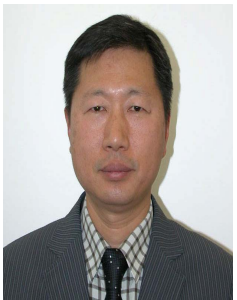

Kisong Yoon received his M.S. and Ph.D degrees in Computer Science from New York City University in 1988 and 1993 respectively. From 1993, he was a principal member of Electronics and Telecommunications Research Institute (ETRI). His research interests are digital contents distribution, digital rights management and digital cinema/signage. 